\title{
TITLE: Sacrificial crystal templated hyaluronic acid hydrogels as biomimetic 3D tissue scaffolds for nerve tissue regenera- tion.
}

\author{
AUTHOR NAMES. Richelle C. Thomas ${ }^{1,2,3}$, Philip Vu ${ }^{3}$, Shan P. Modi ${ }^{2} \ddagger$, Paul E. Chung ${ }^{1,2}+$, R. Clive \\ Landis $^{4}$, Zin. Z. Khaing ${ }^{2,3}$, John G. Hardy ${ }^{2,3, *}$, Christine E. Schmidt ${ }^{2,3, *}$
}

AUTHOR ADDRESS.

1 Mcketta Department of Chemical Engineering, The University of Texas at Austin, Austin, TX, USA; emails: richelle@che.utexas.edu (R.T.); paul.chung@utexas.edu (P.C.).

2 Department of Biomedical Engineering, The University of Texas at Austin, Austin, TX, USA; emails: rich-

elle@che.utexas.edu (R.T.); shanmodi13@gmail.com (S.P.M.), paul.chung@utexas.edu (P.C.),

zinkhaingster@gmail.com (Z.Z.K.); johnhardyuk@gmail.com (J.G.H.).

3 J. Crayton Pruitt Department of Biomedical Engineering, The University of Florida, Gainesville, FL, USA; rich-

elle@che.utexas.edu (R.T.); p.philip.v@gmail.com (P.V.); zinkhaingster@gmail.com (Z.Z.K.);

johnhardyuk@gmail.com (J.G.H.); schmidt@bme.ufl.edu (C.E.S.).

4 Department of Chronic Disease Research Centre, Faculty of Medicine, Cave Hill Campus, The University of the West Indies, Barbados; clandis@uwichill.edu.bb (R.C.L.).

*Authors to whom correspondence should be addressed; J. Crayon Pruitt Department of Biomedical Engineering, The University of Florida, Gainesville, FL 32601; E-mail: (JGH) johnhardyuk@gmail.com; (CES)

schmidt@bme.ufl.edu; Tel (CES):+1-352-273-9222; Fax (CES): +1-352-273-9221

KEYWORDS. hydrogel, sacrificial porogen, pore structure, nerve tissue scaffold.

\begin{abstract}
Pores are key features of natural tissues and the development of tissues scaffolds with biomimetic properties (pore structures and chemical/mechanical properties) offers a route to engineer implantable biomaterials for specific niches in the body. Here we report the use of sacrificial crystals (potassium dihydrogen phosphate or urea) that act as templates to impart pores to hyaluronic acid-based hydrogels. The mechanical properties of the hydrogels were analogous to the nervous system (in the Pascal regime), and we investigated the use of the potassium dihydrogen phosphate crystaltemplated hydrogels as scaffolds for neural progenitor cells (NPCs), and the use of urea crystal-templated hydrogels as scaffolds for Schwann cells. For NPCs cultured inside the porous hydrogels, assays for the expression of Nestin are inconclusive, assays for GFAP and BIII-tubulin expression suggest that the NPCs maintain their undifferentiated phenotype more effectively than the controls (with glial fibrillary acidic protein (GFAP) and BIII-tubulin expression at ca. 50\% relative to the chemically/mechanically equivalent not templated control hydrogels). For Schwann cells cultured within these hydrogels, assays for the expression of S1oo protein or Myelin basic protein confirm the expression of both proteins, albeit at lower levels on the templated hydrogels (ca. 50\%) than on the chemically/mechanically equivalent not templated control hydrogels. Such sacrificial crystal templated hydrogels represent platforms for biomimetic $3 \mathrm{D}$ tissue scaffolds for the nervous system.
\end{abstract}

\section{INTRODUCTION}

Hydrogel-based tissue scaffolds with biomimetic properties (chemical/mechanical properties and pore structures) are potentially useful platforms for both fundamental science and clinical translation, and the development of such hydrogels is therefore an area of intense current research interest in academia and industry. ${ }^{1-6}$ Hydrogels can be manufactured using polymers of natural (i.e. biopolymers) ${ }^{6}$ or synthetic origins (e.g. polyethylene glycol). ${ }^{7}$ The incorporation of naturally occurring components of the extracellular matrix (such as proteins or poly- saccharides) yields hydrogels with chemical properties that to a certain extent instruct the behavior of cells that reside within them. ${ }^{4,5,7}$ It is possible to tune the mechanical properties of hydrogels by controlling the degree of crosslinking of the polymers, where the crosslinks are either physical crosslinks (i.e. chain entanglement) or chemical crosslinks (covalent chain crosslinks [e.g. C-C, S-S linkages] or non-covalent crosslinks [i.e. supramolecular interactions]) ${ }^{1-3}$ It is possible to impart micropores and macropores to biomaterials using a variety of different techniques including the use of sacrificial porogens 
wherein the resultant pore sizes/shapes closely resemble the structure of the porogen, ${ }^{8-11}$ and $3 \mathrm{D}$ printing enables the generation of biomaterials with complex $3 \mathrm{D}$ structures (in some cases including hierarchically structured pores). ${ }^{12-20}$

Realizing the potential of stem cell therapies for regenerative medicine requires the development of robust and reliable materials/methods for expanding stem cells in their undifferentiated state and directing their differentiation towards specific lineages. ${ }^{21-24}$ Stem cell niches are localized regions within native tissue that serve as a repository of lineage-specific stem cells. Engineering biomimetic hydrogels that act as artificial stem cell niches has encouraged investigation of the role of cell-cell and cell-substrate interactions on cell fate based on chemical, mechanical and topographical signals in vitro in both $2 \mathrm{D}$ and ${ }_{3} \mathrm{D}^{25^{-27}}$ The gel structure (mesh sizes, the presence or absence of pores, surface texture, and roughness/topography) has a significant impact on nanoscopic cell-substrate interactions, and macroscopic cellenvironment interactions (diffusion of nutrients/waste, ease of cell infiltration and vascularization). ${ }^{14,28-31}$

Furthermore, the forces that occur when hyaluronic acid (HA) swells are thought to contribute to early neuronal morphogenesis. $^{32}$ Cui et al implanted HA and HA-RGD hydrogels in rat cortical defects, the lyophilized samples had uniform pore size diameters that created a large surface area to volume ratio that accommodated a large number of cells and allowed neovascularization of the matrix. $^{33}$ The HA-RGD hydrogels provided continuity across the defect. Such implants had no visible glial scar formation and were well integrated into the host tissue at 6 weeks; interestingly, HA-hydrogels mitigated glial scar formation, encouraged integration into the host tissue, allowed cell infiltration and angiogenesis while HA-RGD hydrogels encouraged neurite regrowth. ${ }^{33}$ These results suggest that HA-based tissue scaffolds with specific porous structures can control cell-substrate interactions within the respective scaffolds. ${ }^{33}$

NPCs lie in a vascular niche in vivo, ${ }^{34}$ and are multipotent, meaning they have the ability to differentiate into both neurons and glia. ${ }^{34}$ There are three types of glial cells, astrocytes, microglia and oligodendrocytes. The microglia are the resident immune system cells found in the sub ventricular zone of the lateral ventricles and the subgranular zone of the dentate gyrus in adult brains. ${ }^{34}$ These cells have been successfully cultured in threedimensional scaffolds, however with limited viability and proliferation. ${ }^{34}$ Extracellular matrix scaffolds of HA have been used to create bioartificial stem cell niches for endothelial progenitor cells and to improve NPC survival. ${ }^{3}$ In these cases, HA hydrogels were used as a cell delivery medium and did not pertain to phenotype maintenance. There is a market need to expand stem and progenitor cell populations in an undifferentiated state for therapeutic applications. ${ }^{29,35}$ Work that has been conducted on undifferentiated expansion has focused on the interplay of immobilized chemical signaling but not the role of substrate properties for cell maintenance in the undifferenti- ated state. ${ }^{29,35}$ Hydrogels are a very good means of maintaining or differentiating NPCs and research is on-going to determine if hydrogels can be used as a viable means to deliver stem/progenitor cells. ${ }^{29,35}$

Poly(ethyl acrylate)-poly(hydroxyl ethyl acrylate) copolymer blend matrices with two different internal geometries were investigated for their application as implants into the cortical and subventricular zones (SVZ) of the brain. $^{36}$ Such in vitro experiments proved that NPCs readily infiltrated into the scaffold and differentiated into neurons and astrocytes regardless of the specific geometry of the scaffolds. In vivo implants of both parallel cylindrical channels and those with interconnected voids showed local angiogenesis and neurite extension within scaffolds that were implanted in the SVZ, and supported local tissue regeneration. ${ }^{36}$

Neural stem cells undergo polarization and contact guidance responses that can be mimicked through complex structuring of the surface. ${ }^{5}$ The cellular phenotype, cell survival, extension, etc. can be controlled/maximized by varying the elastic modulus of the culture substrate. Soft tissues in vertebrates maintain mechanical properties which are quantified by elastic moduli within specific ranges, which allows distribution and population sorting to occur based on the mechanical properties of the substrate. ${ }^{5}$ Neural cells flourish in softer hydrogels where the mechanical properties more closely match those found in native neural tissues (the modulus of brain tissue is between $0.1-1 \mathrm{kPa},{ }^{37,38}$ and the shear modulus $\mathrm{G}^{\prime}(\mathrm{w})$ oscillatory of adult rat brain is about $330 \mathrm{~Pa})^{39}$ and using materials whose characteristics are similar to brain tissue encourage its successful integration upon implantation. ${ }^{40}$

It is noteworthy that pores are common features of a variety of natural tissues (including bone, muscle, nerve and skin). ${ }^{1,7,12,17,18}$ The generation of biomaterials with biomimetic properties (pore structures and chemical/mechanical properties) offers a route to engineer implantable biomaterials for specific niches in the body, and here we report the results of our investigation of the use of sacrificial crystals ${ }^{10,41}$ as templates of hydrogels for nerve tissue engineering applications.

Hyaluronic acid is a biodegradable polysaccharide that is a component of the extracellular matrix of nervous tissues, and is straightforward to chemically modify to generate photocrosslinkable derivatives, where the mechanical properties can be tuned to closely resemble those of nervous tissues. ${ }^{3}$ The inclusion of sacrificial porogens (potassium dihydrogen phosphate or urea) that crystallize under specific conditions in solutions of photocrosslinkable hyaluronic acid derivatives results in phase separation to polymer-rich and polymer-poor phases. ${ }^{10}$ Photocrosslinking the hyaluronic acid derivative yields a continuous polymer network that allows the sacrificial crystal porogens to be removed by leaching in water, leaving a characteristic pore structure within $2 \mathrm{D}^{10}$ and $3 \mathrm{D}$ hydrogels. ${ }^{41}$ The ability of these materials to act as scaffolds for nerve tissue regeneration are reported herein. 


\section{EXPERIMENTAL SECTION}

Chemicals and Materials. High molecular weight sodium hyaluronate from Streptococcus equi (Sigma 53747), glycidyl methacrylate (Sigma 779342), triethylamine (Sigma To886), hyaluronidase (Sigma H3506) and dextran-FITC of molecular weight 3,00o-5,00o Da (Sigma FD4), Bovine serum albumin (A7906-5oG), phosphate buffered saline $\left(\mathrm{P}_{5493-\mathrm{L}}\right)$, paraformaldehyde, Triton $\mathrm{X}$ 100 (X1oo), Tween 20 and Anti-Myelin Basic Protein (MBP) antibody produced in rabbit (M3821-10oUG) were all purchased from Sigma Aldrich (St Louis, Mo). Antirabbit IgG (whole molecule)-FITC antibody produced in goat (F9887-1ML) and anti-rabbit IgG TRITC produced in goat (T6778) were also purchased from Sigma Aldrich (St Louis, Mo). Urea (Fisher U15), potassium dihydrogen phosphate (Fisher 7778), Acetone (A181), BD PrecisionGlide 25 gauge needles (305122), Luer-Lok syringes (2018-06) and mouse anti-nestin (MA181819) were all purchased from Fisher Scientific. The photoinitiator Irgacure 2959 (55047962) was obtained from Ciba Specialty Chemicals/BASF (Basel, Switzerland). The GRGDLys(FITC) peptide was supplied by American Peptide Company (Product \# 316846) with a 96.7\% peptide purity. DMEM, high glucose (D1383), fetal bovine serum (16000044) and 0.25\% Trypsin-EDTA(1X) Phenol Red (25200114) were purchased from Invitrogen. Anti- $\beta$ III-tubulin produced in mouse (ab7751), anti-GFAP produced in rabbit $\left(\mathrm{ab}_{7260} /\right.$ ab7779) and anti-rabbit Alexa 568 (ab175471) were all purchased from abcam. Anti-nestin clone rate antibodyy (MAB353) was purchased from Millipore. Rabbit anti-S1oo Calcium Binding Protein IgG antibody (FITC) (ABIN728990) was purchased from antibodiesonline.com. All reagents were used as received.

Preparation of Hyaluronic Acid Derivative. The methacrylated hyaluronic acid derivative was prepared in accordance with our previously described methodology, and methacrylation of the primary alcohol was observed for ca. $18 \%$ of the disaccharides in hyaluronic acid as confirmed by ${ }^{1} \mathrm{H}$ NMR, displayed in Figure S1. ${ }^{10,41,42}$

Hydrogel Preparation. Hydrogels were prepared in accordance with our previously described methodology. ${ }^{41}$ In short: hydrogels with $20 \mathrm{mg} / \mathrm{mL}$ methacrylated hyaluronic acid and with $0.3 \%(\mathrm{w} / \mathrm{v})$ photoinitiator (optionally including potassium dihydrogen phosphate [300 $\mathrm{mg} / \mathrm{ml}]$ or urea $[1400 \mathrm{mg} / \mathrm{ml}]$ ) were mixed at ca. $50^{\circ} \mathrm{C}$. The supersaturated polymer solution was injected into disk molds (Grace Biolabs Silicone Isolators \#664301) encased by two glass slides and cooled on ice to cause porogen precipitation within the polymer solution. After crystallization, the polymer was crosslinked around the crystalline network by exposing the hydrogel to $13 \mathrm{~mW} / \mathrm{cm}^{2}$ ultra-violet light. The hydrogels were immersed in deionized water overnight (dilution of 1:10,0oo) to remove the crystalline component, then swollen in $10 \mathrm{mM}$ phosphate-buffered saline for $24 \mathrm{~h}$ (after which mechanical tests were carried out). Optionally, to render the gels cell adhesive (in this study for Schwann cells), a solution of EDC (0.075 mg/ml), NHS (0.075 mg/ml) and GRGDLys(FITC) $(0.075 \mu \mathrm{mol} / \mathrm{ml})$ in sterile deionized water was prepared and gels were im- mersed in these solutions for 2 hours. The liquid was removed and the gels were thoroughly washed with sterile deionized water ( 6 changes of water over 24 hours, dilution of 1:10,0oo), after which cell culture was carried out.

Cryopreservation Scanning Electron Microscopy (CryoSEM). CryoSEM was used to examine the microstructure of the samples. Samples were fixed to the mounting stage with carbon tape, flash frozen in liquid nitrogen (at $-195{ }^{\circ} \mathrm{C}$ ), sublimated, sputter coated with platinum, and imaged using a Zeiss Supra 4oVP SEM.

Progenitor cell differentiation and expansion on templated hydrogels. All animal work was performed in accordance with the Institutional Animal Care and Use Committee at the University of Florida. The forebrains of mice (embryonic day 14, C57BL6, Charles River) were isolated into Hank's Buffered Salt Solution. Using sterile techniques, samples were cut into small pieces using spring scissors, placed in DMEM F12 media with N2 supplement (Gibco), 1\% penicillin/ampicillin/streptomycin solution and dissociated mechanically by pipetting with a fire polished glass Pasteur pipette. About 1 x $10^{6}$ cells were plated in a $10 \mathrm{~cm}$ tissue culture dish and cultured as freefloating cell aggregates or "neurospheres" in the same medium supplemented with $20 \mathrm{ng} / \mathrm{mL}$ basic fibroblast growth factor added every two days. Neurospheres were expanded and used between passage two and five. Not templated hydrogels as well as those templated with potassium dihydrogen phosphate (and not modified with GRGDLys(FITC)) were seeded with 200,00o cells each. The hydrogels were cultured and imaged at 3 and 7 days. The samples were imaged using phase microscopy to observe cells in the gels (analogous to our previous reports, ${ }^{10}$ although the exact penetration depth of the cells in the gels was not determined in this study) and fluorescence microscopy was used to observe markers expressed by the NPCs. Cells were cultured in serum-free DMEM F12 HAM media with $1 \% \mathrm{~N}_{2}$ supplement (Gibco) and $1 \%$ penicillinstreptomycin with basic fibroblast growth factor and epidermal growth factor at $20 \mathrm{ng} / \mathrm{mL}$ media to encourage expansion but not differentiation of the cell population in the experiment. Control experiments: tissue culture plates were used as the positive control, not templated hydrogels were used as the experimental control, templated hydrogels with $10 \%$ DMSO in the media were used as the negative control. At the end of the experiment, cells were fixed with $4 \%$ paraformaldehyde and stained with primary antibodies against nestin (undifferentiated progenitor cells, Millipore), $\beta$-III tubulin (neuronal marker, Abcam), GFAP (glial marker, Abcam) then fluorescent secondary antibodies prior to imaging. Cells were counterstained with DAPI to label all nuclei. The cell culture media was gently replaced every two days. Z-stack images were taken using a Zeiss Superscope to visualize the apparent cell types within the hydrogel. Each experimental condition was performed in triplicate. The images were taken using the same microscope under the same settings. The images were subsequently analyzed for intensity in each color channel and normalized to the intensity for the number of cells (DAPI staining). 
Schwann cell culture at various Forskolin (FSK) concentrations in culture and on hydrogels. Rat Schwann cells were purchased frozen from ATCC (CRL2765) and cultured in DMEM with $10 \%(\mathrm{w} / \mathrm{v})$ fetal bovine serum. The cell line was first expanded then cultured in media with bovine pituitary extract and either o $\mu \mathrm{M}$, o.o1 $\mu \mathrm{M}$ or $10 \mu \mathrm{M}$ FSK, either on tissue culture plastic or on the hydrogels modified with GRGDLys(FITC) with 250,000 cells. The cells were cultured for 48 hours prior to analysis. For imaging, cells were first rinsed in warm PBS then fixed with $4 \%$ paraformaldehyde for 15 minutes. The cells were permeabilized with $0.2 \%$ Triton-Xıo in PBS for 30 minutes. The cells were stained with anti-Sioo (FITC) at 1:500 for one hour, then rinsed three times. Next the cells were incubated with anti-myelin basic protein raised in rabbit at 1:500 for one hour, rinsed three times and incubated with anti-rabbit Alexa 568 for one hour. After rinsing the cell nuclei were stained with DAPI (1:10oo). Cells that were in the hydrogels were stained at the same concentrations but the antibodies were allowed to incubate overnight followed by 6 hour rinses.

For flow cytometry, the cells cultured on tissue culture plastic were incubated with $0.25 \%$ Trypsin-EDTA to remove the cells. Cells were analyzed at a concentration of 150,000 cells per vial $(n=10)$. The same permeabilization, staining and wash steps took place, however they were conducted on ice and antibodies were incubated for 20 minutes. For flow cytometry, anti-Sioo(FITC) was used at 1:10o, rabbit anti-myelin basic protein primary antibody at 1:10o and anti-rabbit IgG-TRITC produced in goat at 1:10o. Hydrogels were digested in hyaluronidase to remove cells prior to flow cytometry that was conducted using a Beckman Coulter(R) EPICS XL-MCL Acquisition Flow Cytometer, Class 1 Laser.

\section{RESULTS AND DISCUSSION}

Hydrogel preparation and characterization. The aim of this study was to investigate the hyaluronic acidbased hydrogels to assess their potential application in the regeneration of the nervous system. We employed a simple method of imparting macropores to the bulk and surface of hyaluronic acid-based hydrogels using sacrificial crystal templates, ${ }^{10,}{ }^{41}$ and studied the morphologies of samples of the batch of hydrogels used in this study by SEM.

CryoSEM images reveal the surface of not templated hydrogels to be rough but not macroporous (Figure $1 \mathrm{~A}$ ), whereas the potassium dihydrogen phosphate crystal templated hydrogels had starburst-like voided areas of 50$100 \mu \mathrm{m}$ width throughout the volume of the hydrogel (Figure $1 \mathrm{~B}$ ), and the urea crystal templated hydrogels exhibited void spaces pores with lengths/widths of greater than $10 \mu \mathrm{m}$ throughout the hydrogel with some evidence of urea crystal alignment ${ }^{10,41}$ in the $\mathrm{x}-\mathrm{y}$ dimension (Figure 1 C), and in both cases, the pores imparted to the hydrogels by the sacrificial porogens should be cell-permeable by virtue of their size.
The bulk mechanical properties of hydrogel scaffolds play a role in their performance in vivo, and rheological experiments show that the storage modulus, G', of not templated gels was ca. $100 \mathrm{~Pa}$., by comparison with $0.1 \mathrm{~Pa}$ for urea templated gels or o.6-8.5 $\mathrm{Pa}$ or potassium dihydrogen phosphate templated gels, respectively; ${ }^{41}$ and therefore that templation offers a method of tuning the mechanics of the gels to suit a specific biological niche. ${ }^{41}$ Moreover, we have previously shown that the not templated and templated hydrogels were non-cytotoxic towards fibroblasts and Schwann cells in vitro. ${ }^{41}$
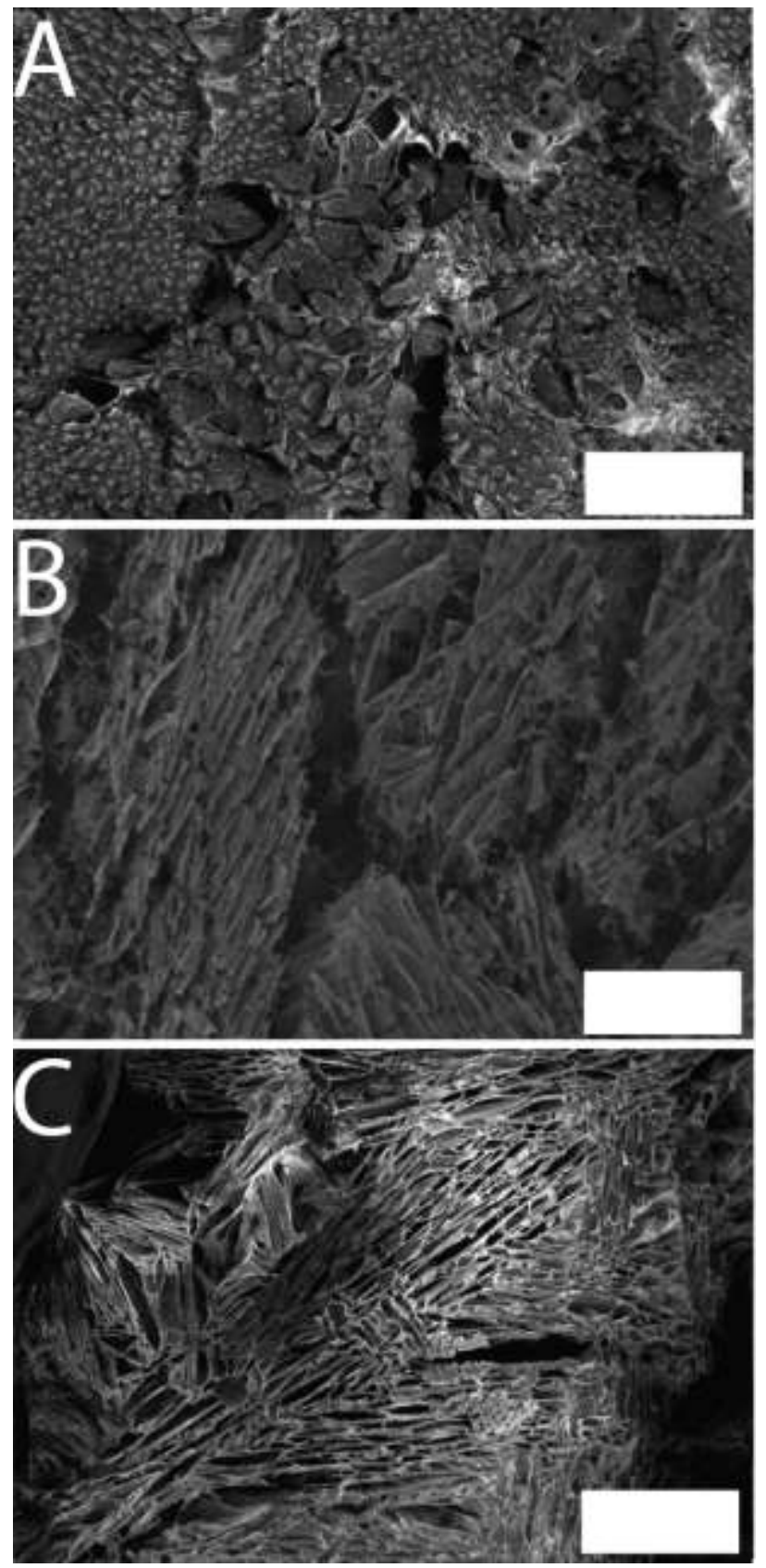

Figure 1. SEM images of hydrogel architecture. A) Not templated hydrogels. Scale bar represents $200 \mu \mathrm{m}$. B) Potassium dihydrogen phosphate templated hydrogels. Scale bar represents $200 \mu \mathrm{m}$. C) Urea templated hydrogels. Scale bar represents $200 \mu \mathrm{m}$. 
NPCs are particularly interesting for neural tissue engineering applications, and with a view to the use of the hydrogels for such applications we investigated the growth of NPCs within the starburst-like voids in the potassium dihydrogen phosphate-templated hydrogels. By comparison, the needle-like pores observed within the urea-templated hydrogels ${ }^{10,41}$ are similar to the directional pores found in the peripheral nervous system and such hydrogels were used to culture Schwann cells.

NPC culture studies. The potassium dihydrogen phosphate-templated hydrogels were effective in allowing NPCs to grow within the void spaces. Images of NPCs cultured for 3 days are shown in Figure 2 and those cultured for 7 days are shown in Figures 3 and 4, and data for the expression of $\beta$ III-tubulin and Nestin are displayed in Figure 5.

NPCs cultured for 3 days in vitro in suspension are shown in Figure $2 \mathrm{~A}-\mathrm{D}$, and those cultured in the hydrogels are shown in Figure 2 E-H. The NPCs cultured in the not templated hydrogels expressed higher amounts of nestin at 3 days in culture (Figure $2 \mathrm{E}$ and $\mathrm{F}$ ) than those NPCs cultured in suspension (Figure $2 \mathrm{~A}$ and B) and indeed those NPCs cultured in the potassium dihydrogen phosphate-templated hydrogels (Figure $2 \mathrm{G}$ and $\mathrm{H}$ ) where evidence of neurosphere formation after 3 days was also evident. In regards to glial fibrillary acidic protein (GFAP) expression, after 3 days in culture, the not templated samples have the lowest GFAP expression. After 7 days, cells cultured in either not templated or templated hydrogels have relatively more GFAP expression, but the cells in suspension in medium containing $10 \%$ DMSO have the highest (Figure $2 \mathrm{C}$ and $\mathrm{D}$ ).

NPCs cultured for 7 days in vitro in suspension are shown in Figure $3 \mathrm{~A}-\mathrm{D}$, and those cultured in the hydrogels are shown in Figure $3 \mathrm{E}-\mathrm{H}$. At both 3 and 7 days the nestin expression in not templated samples is either comparable to or slightly higher than the cell suspension controls. The NPCs in suspension formed neurospheres after 7 days with some minor evidence of differentiation (Figure $3 \mathrm{~A}$ and $\mathrm{B}$ ). We observed minor neuronal and astrocytic differentiation after 7 days in not templated hydrogels (Figure $3 \mathrm{E}$ and $\mathrm{F}$ ) and neuronal differentiation in the potassium dihydrogen phosphate-templated hydrogels (Figure $3 \mathrm{G}$ and $\mathrm{H}$ ). We observed both neuronal and astrocytic differentiation after 7 days in culture in suspension in medium containing $10 \%$ DMSO (Figure $3 \mathrm{C}$ and D).

NPCs that have maintained their undifferentiated phenotype express more nestin, and while the relative fluorescence measurements (Figure 5) may suggest that the templated gels performed less well than cells in suspension and not templated hydrogels, our assays of BIIItubulin (Figure 5) that is also a sign of differentiation potential suggest that this is not the case. In each case, cells in suspension as well those on/in both not templated and templated hydrogels expressed lower levels of differentiation markers than cells cultured in the negative control samples with cells in suspension in medium containing $10 \%$ DMSO.

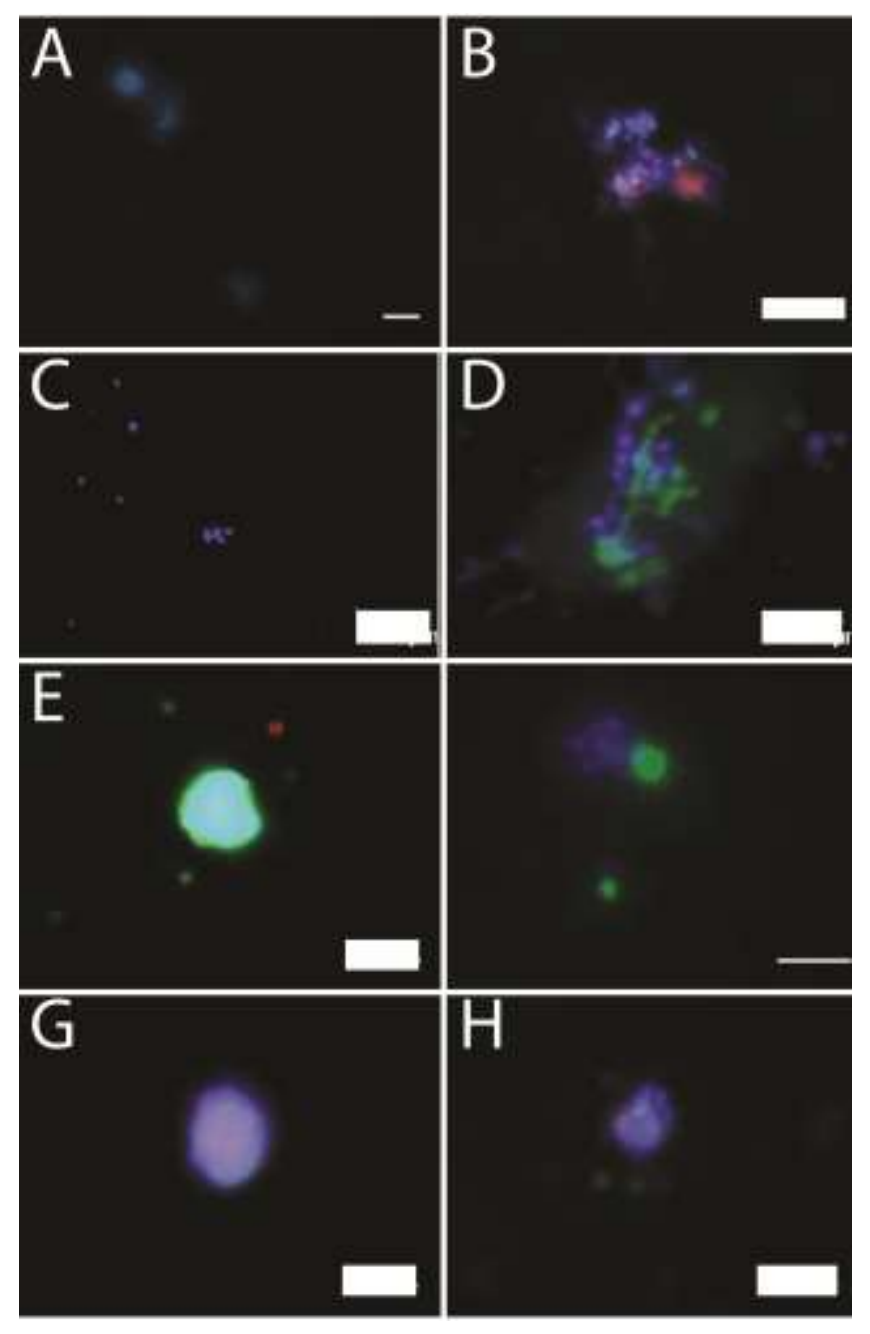

Figure 2. NPCs cultured for 3 days in vitro in suspension (AD) and on/in hydrogels (E-H). A) Cells in suspension. Stained with DAPI (blue), nestin (green) and BIII-tubulin (red). Scale bar represents $50 \mu \mathrm{m}$. B) Cells in suspension. Stained with DAPI (blue), nestin (green) and GFAP (red). Scale bar represents $50 \mu \mathrm{m}$. C) Cells in suspension in medium with 10\% DMSO. The cells were stained with DAPI (blue), nestin (green) and BIII-tubulin (red, left). Scale bar represents $100 \mu \mathrm{m}$. D) Cells in suspension in medium with $10 \%$ DMSO. The cells were stained with DAPI (blue), nestin (green) and GFAP (red, right). Scale bar represents $100 \mu \mathrm{m}$. E) Not templated control hydrogels. Stained with DAPI (blue), nestin (green) and BIII-tubulin (red). Scale bar represents $50 \mu \mathrm{m}$. F) Not templated control hydrogels. Stained with DAPI (blue), nestin (green) and GFAP (red). Scale bar represents $50 \mu \mathrm{m}$. G) Potassium dihydrogen phosphatetemplated hydrogels. Stained with DAPI (blue), nestin (green) and BIII-tubulin (red). Scale bar represents $50 \mu \mathrm{m} . \mathrm{H}$ ) Potassium dihydrogen phosphate-templated hydrogels. Stained with DAPI (blue), nestin (green) and GFAP (red). Scale bar represents $50 \mu \mathrm{m}$. 


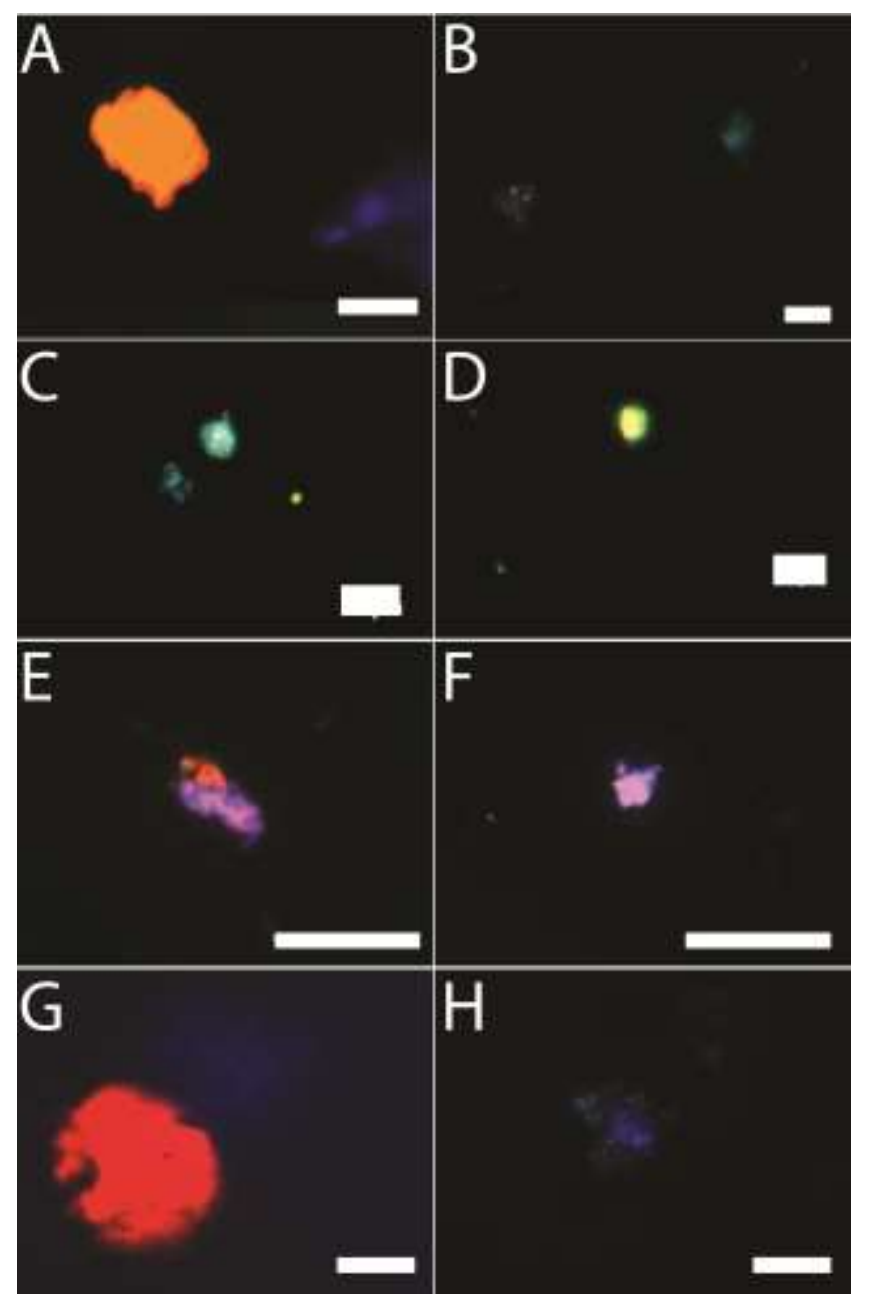

Figure 3. NPCs cultured for 7 days in vitro in suspension (AD) and on/in hydrogels (E-H). A) Cells in suspension. Stained with DAPI (blue), nestin (green) and BIII-tubulin (red). Scale bar represents $50 \mu \mathrm{m}$. B) Cells in suspension. Stained with DAPI (blue), nestin (green) and GFAP (red). Scale bar represents $50 \mu \mathrm{m}$. C) Cells in suspension in medium with 10\% DMSO. Stained with DAPI (blue), nestin (green) and BIII-tubulin (red). Scale bar represents $50 \mu \mathrm{m}$. D) Cells in suspension in medium with 10\% DMSO. Stained with DAPI (blue), nestin (green) and GFAP (red). Scale bar represents $50 \mu \mathrm{m}$. E) Not templated control hydrogels. Stained with DAPI (blue), nestin (green) and BIII-tubulin (red). Scale bar represents $50 \mu \mathrm{m}$. F) Not templated control hydrogels. Stained with DAPI (blue), nestin (green) and GFAP (red). Scale bar represents $50 \mu \mathrm{m}$. G) Potassium dihydrogen phosphate-templated hydrogels. Stained with DAPI (blue), nestin (green) and BIII-tubulin (red). H) Potassium dihydrogen phosphate-templated hydrogels. Stained with DAPI (blue), nestin (green) and GFAP (red, right).

Microscopy of NPCs cultured for 7 days in vitro in potassium dihydrogen phosphate-templated templated hydrogels at 10x and 2ox magnification with respective hemocytometer images are shown in Figure 4 A-D, showing inclusion of the NPCs in the void spaces left by the sacrificial porogen.
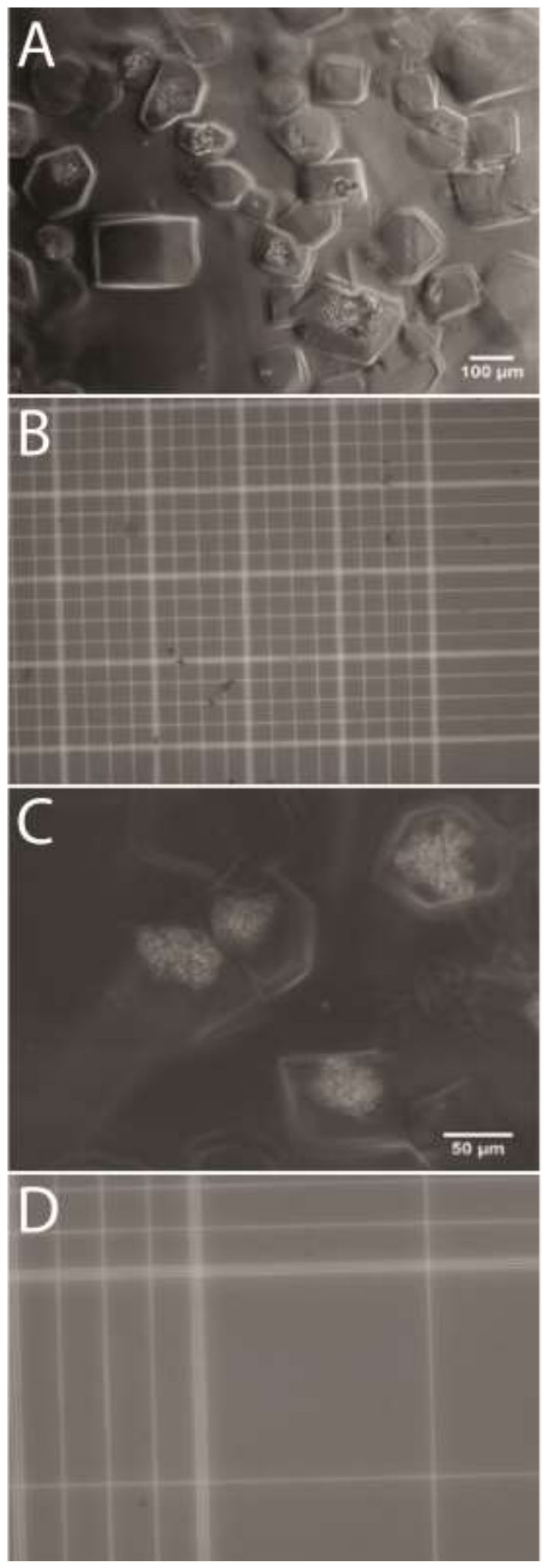

Figure 4. Microscope images. A) NPCs cultured for 7 days in vitro in potassium dihydrogen phosphate-templated templated hydrogels at 1ox magnification. B) Hemocytometer at 10x magnification. C) NPCs cultured for 7 days in vitro in potassium dihydrogen phosphate-templated templated hydrogels at 20x magnification. D) Hemocytometer at 20x magnification. 


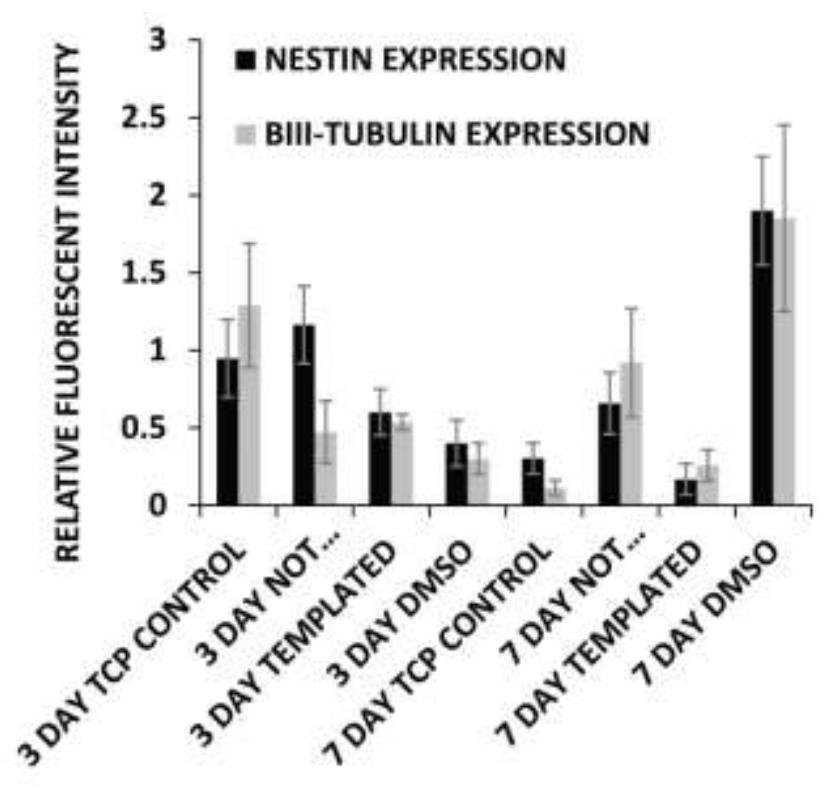

Figure 5. Data for the expression of BIII-tubulin and Nestin. Relative fluorescence intensity of nestin and ßIII-tubulin expression from E11.5 forebrain progenitor cells.

Schwann cell culture studies. Schwann cells do not adhere strongly to HA, and therefore a cell adhesive peptide (GRGDLys(FITC)) was conjugated to the HA to render the resulting hydrogels adhesive towards Schwann cells (or indeed PC12 cells). ${ }^{43}$ Schwann cells secrete a sheath of Myelin that allows for improved conduction along axons in the peripheral nervous system. As immature Schwann cells come in contact with axons, the Schwann cells are activated to wrap around the nerves and form a compact, insulting sheath around the nerve fibers. Myelination in the peripheral nervous system by Schwann cells differs from that of the oligodendrocytes in the central nervous system. Schwann cells wrap around axons in a one to one fashion, however oligodendrocytes myelinate several nerve fibers at once to form a bundle. During the "active myelination period", which extends through the second, third and fourth weeks after birth, myelin markers such as myelin protein zero and myelin basic protein are expressed. ${ }^{44}$

Literature suggests that materials used to shape the microenvironment can also be used to engineer the immune response, by the use of cytokine-releasing scaffolds. ${ }^{45,46}$ Schwann cells were cultured in a pro-myelinating media and their phenotype verified with myelin basic protein markers. Cells were evaluated for changes in myelin basic protein (MBP) after 48 hours using flow cytometry (Figure 6). ${ }^{47}$

S1oo or MBP down/upregulation correlates to the degree of Schwann-like behavior the cell population expresses. A population of Schwann cells in culture is susceptible to a proportion of the number being fibroblasts that do not express Sioo (consequently, a higher percentage of the population expressing Sioo signifies a higher degree of Schwann cell purity within the population). For experiments that require Schwann cell phenotype, this is a positive outcome. Moreover, the expression of MBP by a
Schwann cell population confirms that they are mylenating Schwann cells (a positive outcome for studies aiming to induce a mylening state within the cultured population).

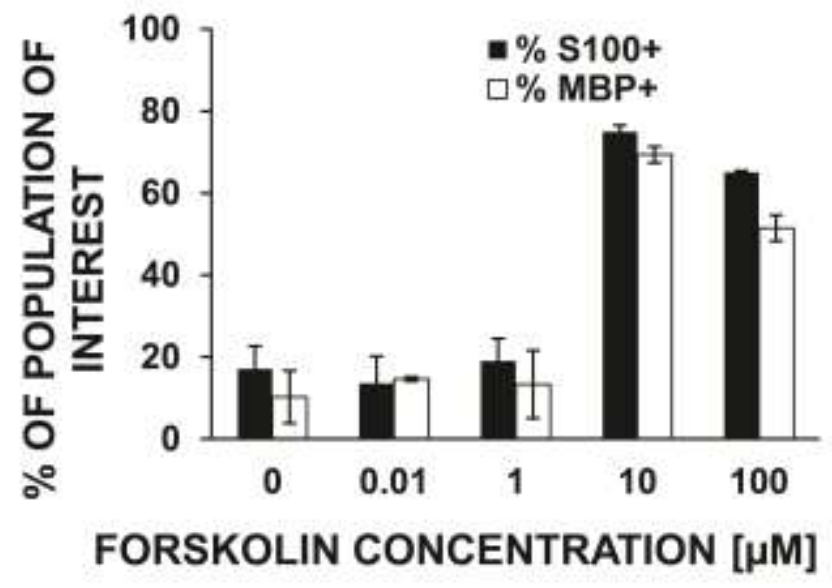

Figure 6. Dose-Dependent Myelin Production in Schwann Cells. Schwann cells cultured with varying FSK concentrations and flow cytometry performed on population. Increase occurs at $10 \mu \mathrm{M}$ FSK where the percentage of the population expressing Sioo and myelin basic protein increases. Forskolin is a cyclicAMP activator so it is possible that the high levels of FSK induced an increase in the percentage of the cell population that was S1oo+ and the percentage that expressed MBP.

The flow cytometry results were used to provide qualitative measures of the levels of myelin markers present in the culture population. Schwann cell cultures may be contaminated by fibroblasts, therefore a parallel culture of Schwann cells was maintained and stained for Sioo to determine the purity of the culture population. The cells were cultured in media containing varying concentrations of FSK which is a cyclic AMP activator and therefore can be used to artificially induce myelin expression from Schwann cells. The proteins were analyzed independently so the percentages presented are based on the total cell population (Figure 6). 

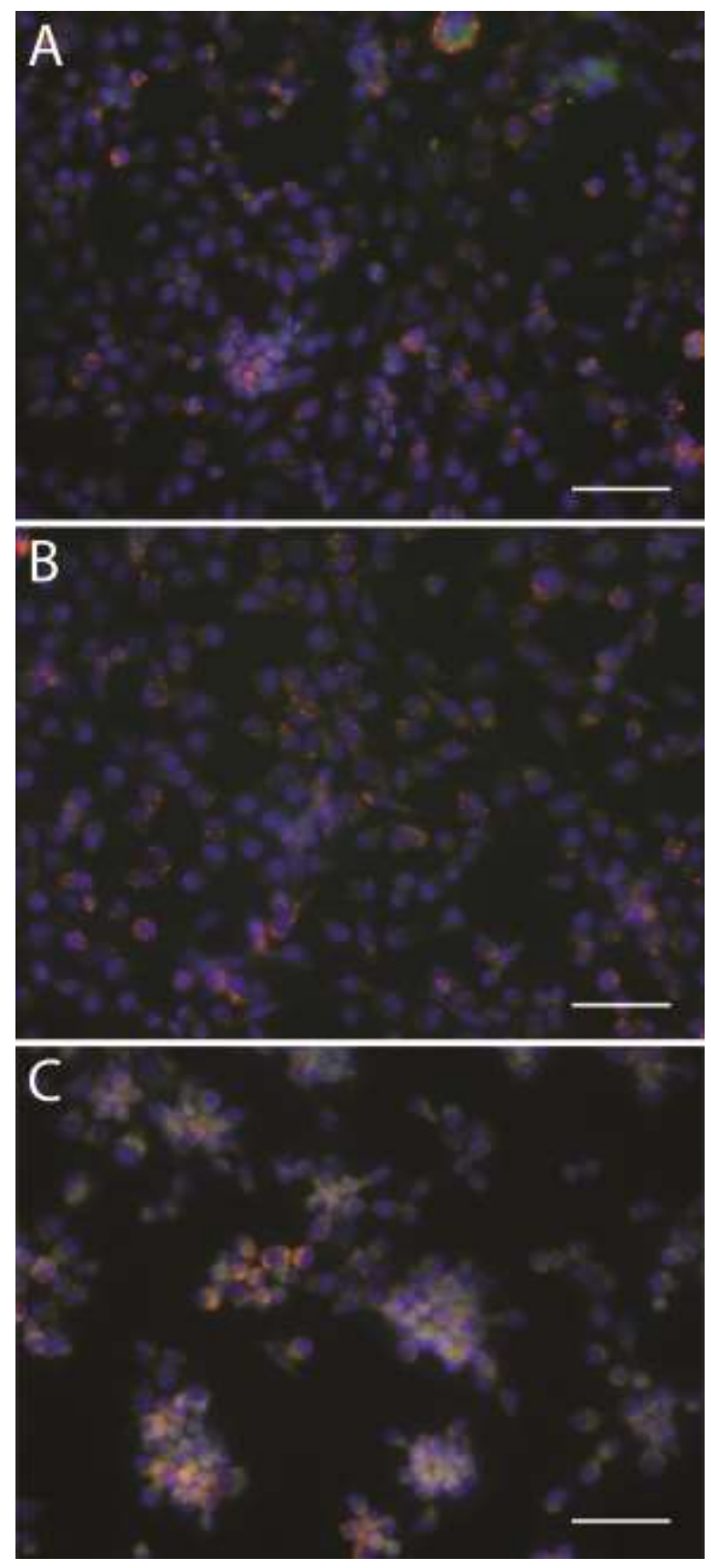

Figure 7. Schwann cells cultured for 48 hours in varying concentrations of FSK. A) Cells were cultured in o $\mu$ M FSK. Scale bar represents $50 \mu \mathrm{m}$. B) Cells were cultured in o.o1 $\mu \mathrm{M}$ FSK. Scale bar represents $50 \mu \mathrm{m}$. C) Cells were cultured in $10 \mu \mathrm{M}$ FSK. Scale bar represents $50 \mu \mathrm{m}$. Cells were stained for DAPI (blue), Sioo (green), and myelin basic protein (red).

Further experiments conducted with $\mathrm{o} \mu \mathrm{M}$, o.o1 $\mu \mathrm{M}$ and $10 \mu \mathrm{M}$ FSK were carried out. Cells were then seeded on templated and not templated scaffolds and again evaluated for phenotype retention after 48 hours in culture with the potential to magnify the effect of FSK. Fluorescent images of the cells in the $o \mu \mathrm{M}, 0.01 \mu \mathrm{M}, 1 \mu \mathrm{M}$ and $10 \mu \mathrm{M}$ FSK are shown in Figure 7, where the cell nuclei were stained with DAPI (blue), cells that express the S1oo protein were labeled with FITC (green) and myelin basic protein was labeled with Alexa 568 (red).
There appears to be an increase in the myelin basic protein expression in both the cells cultured in the presence of $0.01 \mu \mathrm{M}$ and $10 \mu \mathrm{M}$ FSK as compared to the $o \mu \mathrm{M}$ control. There also appears to be an upregulation of S1oo in the cells cultured in $10 \mu \mathrm{M}$ FSK as evidenced by the green and red co-localization. Figure 8 confirms the phenomena where a significant increase in both S1oo and MBP occur with $10 \mu \mathrm{M}$ FSK by flow cytometry analysis. These results indicate that culture in the presence of high levels of the FSK cyclic AMP activator yields increased numbers of Sioo+ Schwann-like cells, either because the fibroblast like cells do not survive as well, or because the Schwann cells proliferate faster.

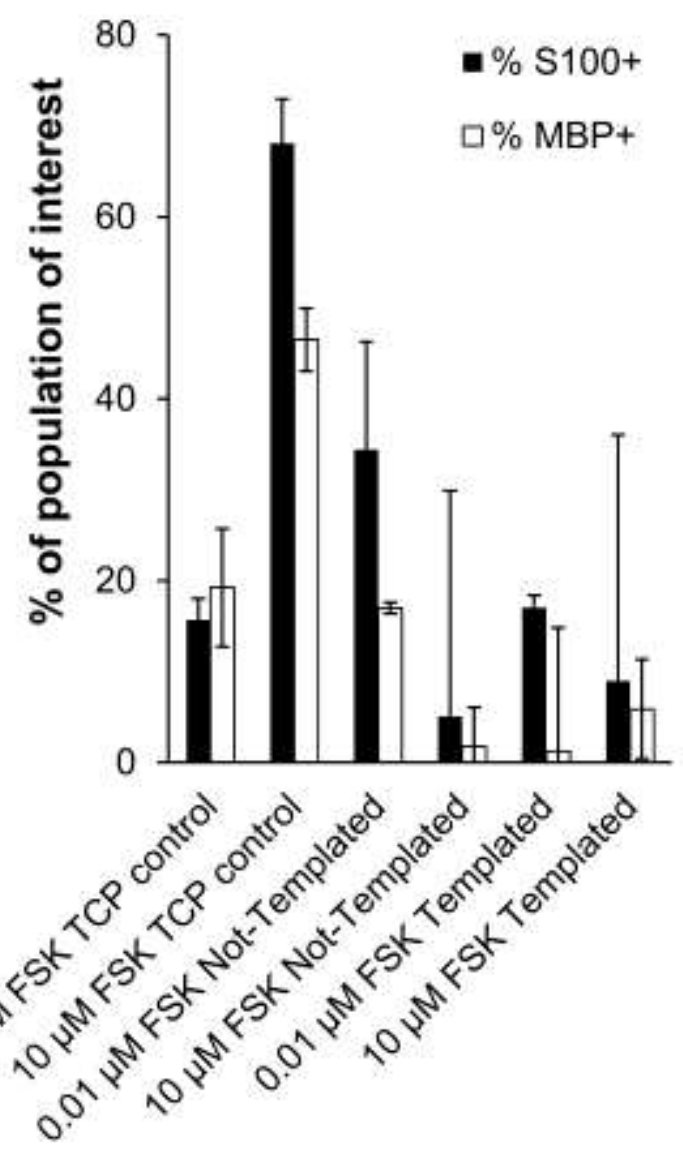

Figure 8. Dose-dependent myelin production in Schwann cells cultured within urea-templated hydrogels.

The cells were also cultured on/in not templated control hydrogels and urea-templated scaffolds at $0.01 \mu \mathrm{M}$ and $10 \mu \mathrm{M}$ FSK concentrations in media (Figure 8). For the cell culture plastic control samples, the percentage of the population expressing both S1oo and MBP increased with increasing FSK concentration. However, when the cells were cultured on/in hydrogels, this trend was not observed. In both the not templated and urea-templated samples the S1oo and MBP expression was reduced both relative to the cell culture plastic controls and the cells cultured in the presence of o.01 to $10 \mu \mathrm{M}$ FSK.

\section{CONCLUSIONS}


The development of biomimetic tissue scaffolds is a promising avenue of research for tissue engineering, and pore structure is an important feature of natural tissues. A simple, bench-top process using cheap sacrificial crystal templates was adapted ${ }^{10,41}$ to create complex architecture within three-dimensional photopolymerized hydrogels that can be easily handled in the lab yielding a useful platform for in vitro studies of pore architecture on cellular behavior. We believe that rational use of a bioactive molecule as a sacrificial crystal template could serve a secondary role as therapeutic agents ${ }^{48}$ delivered over a sustained period of time, yielding tissue scaffolds that instruct cell behavior not only because of their architectures, but also the action of the bioactive molecule. The architecturally complex templated hydrogels described here each hold promise for $3 \mathrm{D}$ cell culture environments, potentially for the regeneration of the nervous system.

\section{ASSOCIATED CONTENT}

Supporting Information. ${ }^{1} \mathrm{H}$ NMR of the nonphotocrosslinked conjugate is displayed in Figure S1. "This material is available free of charge via the Internet at http://pubs.acs.org."

\section{AUTHOR INFORMATION}

\section{Corresponding Author}

* Authors to whom correspondence should be addressed; J. Crayon Pruitt Department of Biomedical Engineering, The University of Florida, Gainesville, FL 326o1; E-mail: (JGH) johnhardyuk@gmail.com; (CES) schmidt@bme.ufl.edu; Tel (CES):+1-352-273-9222; Fax (CES): +1-352-273-9221.

\section{Author Contributions}

The manuscript was written through contributions of all authors. All authors have given approval to the final version of the manuscript. $₫$ These authors contributed equally.

\section{Funding Sources}

National Science Foundation Division of Materials Research Grant 0805298 was used to support the research described in the manuscript. The National GEM Consortium and generous startup resources supplied by the University of Florida also provided funds to support the research described in the manuscript.

\section{ACKNOWLEDGMENT}

We thank Dr. Leandro Forciniti and Dr. Sarah Mayes at the University of Texas at Austin, and Dr. Clive Landis at the University of the West Indies, Cave Hill in Barbados, West Indies for informative discussions.

\section{ABBREVIATIONS}

NMR, Nuclear Magnetic Resonance. SEM, Scanning Electron Microscopy.

\section{REFERENCES}

(1) Slaughter, B.V.; Khurshid, S.S.; Fisher, O.Z.; Khademhosseini, A.; Peppas. N.A. Hydrogels in regenerative medicine. Adv. Mater. 2009, 21, 3307-3329.
(2) Van Vlierberghe, S.; Dubruel, P.; Schacht, E. BiopolymerBased Hydrogels As Scaffolds for Tissue Engineering Applications: A Review. Biomacromol. 2011, 12, 1387-1408.

(3) Burdick, J.A.; Prestwich, G.D. Hyaluronic acid hydrogels for biomedical applications. Adv. Mater. 2011, 23, $\mathrm{H}_{41}-\mathrm{H}_{5} 6$.

(4) Patterson, J.; Martino, M.M.; Hubbell, J.A. Biomimetic materials in tissue engineering. Materials Today 2010, 13, 14-22.

(5) Khaing, Z.Z.; Thomas, R.C.; Geissler, S.A.; Schmidt, C.E. Advanced biomaterials for repairing the nervous system: what can hydrogels do for the brain? Materials Today. 17 (7), 332-340.

(6) Mano, J.F.; Silva, G.A.; Azevedo, H.S.; Malafaya, P.B.; Sousa, R.A.; Silva, S.S.; Boesel, L.F.; Oliveira, J.M.; Santos, T.C.; Marques, A.P.; Neves, N.M. Reis, R.L. Natural origin biodegradable systems in tissue engineering and regenerative medicine: present status and some moving trends. J. Roy. Soc. Int. 2007, 4, 999-1030.

(7) Zhu, J.; Marchant, R.E. Design properties of hydrogel tissue-engineering scaffolds. Expert Rev. Med. Devices. 2011, 8, 6o7-626.

(8) Chiu, Y.C.; Larson, J.C.; Isom, A.Jr.; Brey, E.M. Generation of porous poly(ethylene glycol) hydrogels by salt leaching. Tissue Eng Part C Methods. 2010, 16, 905-12.

(9) Lanasa, S.M.; Hoffecker, I.T.; Bryant, S.J. Presence of pores and hydrogel composition influence tensile properties of scaffolds fabricated from well-defined sphere templates. J. Biomed. Mater. Res. B Appl. Biomater. 2011, 96, 294-302.

(10) Zawko, S.A.; Schmidt, C.E. Crystal templating dendritic pore networks and fibrillar microstructure into hydrogels. Acta Biomater. 6 (2010) 2415-21.

(11) Hardy, J.G.; Geissler, S.A.; Aguilar, D.; Villancio-Wolter, M.K.; Mouser, D.J.; Sukhavasi, R.C.; Cornelison, R.C.; Tien, L.W.; Preda, R.C.; Hayden, R.S.; Chow, J.K.; Nguy, L.; Kaplan, D.L.; Schmidt, C.E. Instructive Conductive $3 \mathrm{D}$ Silk Foam-Based Bone Tissue Scaffolds Enable Electrical Stimulation of Stem Cells for Enhanced Osteogenic Differentiation. Macromol. Biosci., 2015, 15, 1490-1496.

(12) Warner, J.; Soman, P.; Zhu, W.; Tom, M.; Chen, S. Design and ${ }_{3} \mathrm{D}$ Printing of Hydrogel Scaffolds with Fractal Geometries. ACS Biomater. Sci. Eng., 2016, 2 (10), pp 1763-1770.

(13) Bajaj, P.; Schweller, R.M.; Khademhosseini, A.; West, J.L.; Bashir, R. 3D Biofabrication Strategies for Tissue Engineering and Regenerative Medicine. Annu. Rev. Biomed. Eng. 2014, 16, 247-276.

(14) Annabi, N.; Nichol, J.W.; Zhong, X.; Ji, C.; Koshy, S.; Khademhosseini, A.; Dehghani, F. Controlling the Porosity and Microarchitecture of Hydrogels for Tissue Engineering. Tissue Eng Part B Rev. 2010 Aug; 16(4): 371-383.

(15) Guvendiren, M.; Molde, J.; Soares, R.M.D.; Kohn, J. Designing Biomaterials for $3 \mathrm{D}$ Printing. ACS Biomater. Sci. Eng., 2016, 2 (10), pp 1679-1693.

(16) Jose, R.R.; Rodriguez, M.J.; Dixon, T.A.; Omenetto, F.; Kaplan, D.L. Evolution of Bioinks and Additive Manufacturing Technologies for 3D Bioprinting. ACS Biomater. Sci. Eng., 2016, 2 (10), pp 1662-1678.

(17) Jang, J.; Yi, H.-G.; Cho, D.-W. 3D Printed Tissue Models: Present and Future. ACS Biomater. Sci. Eng., 2016, 2 (10), pp 1722-1731.

(18) Ratheesh, G.; Venugopal, J.R.; Chinappan, A.; Ezhilarasu, H.; Sadiq, A.; Ramakrishna, S. 3D Fabrication of Polymeric Scaffolds for Regenerative Therapy. ACS Biomater. Sci. Eng., DOI: 10.1021/acsbiomaterials.6boo37o.

(19) Ouyang, L.; Highley, C.B.; Rodell, C.B.; Sun, W.; Burdick, J.A. 3D Printing of Shear-Thinning Hyaluronic Acid Hydrogels with Secondary Cross-Linking. ACS Biomater. Sci. Eng., 2016, 2 (10), 1743-1751.

(20) Torres-Rendon, J.G.; Femmer, T.; De Laporte, L.; Tigges, T.; Rahimi, K.; Gremse, F.; Zafarnia, S.; Lederle, W.; Ifuku, S.; 
Wessling, M.; Hardy, J.G.; Walther, A. Bioactive gyroid scaffolds formed by sacrificial templating of nanocellulose and nanochitin hydrogels as instructive platforms for biomimetic tissue engineering. Adv. Mater. 2015, 27, 2989-2995.

(21) Guilak, F.; Cohen, D.M.; Estes, B.T.; Gimble, J.M.; Liedtke, W.; Chen, C.S. Control of stem cell fate by physical interactions with the extracellular matrix. Cell Stem cell, 2009, 5, 17-26.

(22) Martínez, E.; Lagunas, A.; Mills, C.A.; Rodríguez-Seguí, S.; Estévez, M.; Oberhansl, S.; Comelles, J.; Samitier, J. Stem cell differentiation by functionalized micro-and nanostructured surfaces. Nanomedicine, 2009, 4, 65-82.

(23) Murtuza, B.; Nichol, J.W.; Khademhosseini, A. Micro-and nanoscale control of the cardiac stem cell niche for tissue fabrication. Tiss. Eng. Part B: Reviews, 2009, 15, 443-454.

(24) Kshitiz; Park, J.; Kim, P.; Helen, W.; Engler, A.J.; Levchenko, A., Kim, D.H. Control of stem cell fate and function by engineering physical microenvironments. Integr. Biol. 2012, 4, 1008-18.

(25) Dellatore, S.M.; Garcia, A.S.; Miller, W.M. Mimicking stem cell niches to increase stem cell expansion. Curr. Opin. Biotechnol. 2008, 19, 534-540.

(26) Moore, K.A.; Lemischka, I.R.; Stem cells and their niches. Science, 2006, 311, 1880-1885.

(27) Lutolf, M.P.; Blau, H.M.; Artificial stem cell niches. Adv. Mater., 2009, 21, 3255-3268.

(28) Lutolf, M.; Hubbell, J. Synthesis and physicochemical characterization of end-linked poly (ethylene glycol)-co-peptide hydrogels formed by Michael-type addition. Biomacromolecules, 2003, 4, 713-722.

(29) Woerly, S., Hydrogels for neural tissue reconstruction and transplantation. Biomaterials, 1993, 14, 1056-1058.

(30) Whang, K.; Healy, K.E.; Elenz, D.R.; Nam, E.K.; Tsai, D.C.; Thomas, C.H.; Nuber, G.W.; Glorieux, F.H.; Travers, R.; Sprague, S.M. Engineering bone regeneration with bioabsorbable scaffolds with novel microarchitecture. Tissue engineering, 1999. 5(1): p. 35-51.

(31) Pettikiriarachchi, J.T.; Parish, C.L.; Shoichet, M.S.; Forsythe, J.S.; Nisbet, D.R. Biomaterials for brain tissue engineering. Aus. J. Chem. 2010, 63, 1143-1154.

(32) Albersdörfer, A.; Sackmann, E. Swelling behavior and viscoelasticity of ultrathin grafted hyaluronic acid films. Eur. Phys. J. B. 1999, 10, 663-672.

(33) Cui, F.; Tian, W.M.; Hou, S.P.; Xu, Q.Y.; Lee, I.S. Hyaluronic acid hydrogel immobilized with RGD peptides for brain tissue engineering. J. Mater. Sci. Mater. Med. 2006, 17, 1393-1401.

(34) Shen, Q.; Goderie, S.K.; Jin, L.; Karanth, N.; Sun, Y.; Abramova, N.; Vincent, P.; Pumiglia, K.; Temple, S. Endothelial cells stimulate self-renewal and expand neurogenesis of neural stem cells. Science, 2004, 304, 1338-1340.

(35) Ren, Y.-J.; Zhou, Z.-Y.; Cui, F.; Wang, Y.; Zhao, J.; Xu, Q. Hyaluronic acid/polylysine hydrogel as a transfer system for transplantation of neural stem cells. J. Bioact. Comp. Polym., 2009, 24, 56-62.

(36) Martinez-Ramos, C.; Vallés-Lluch, A.; Verdugo, J.M.; Ribelles, J.L.; Barcia Albacar, J.A.; Orts, A.B.; Soria López, J.M.; Pradas, M.M. Channeled scaffolds implanted in adult rat brain. J. Biomed. Mater. Res. A, 2012, 100, 3276-3286.

(37) Engler, A.J.; Sen, S.; Sweeney, H.L.; Discher, D.E. Matrix Elasticity Directs Stem Cell Lineage Specification. Cell. 2006, 126, 677-689.

(38) Toepke, M.W.; Impellitteri, N.A.; Theisen, J.M.; Murphy, W.L. Characterization of Thiol-Ene Crosslinked PEG Hydrogels. Macromol. Mater. Eng. 2013, 298, 699-703.

(39) Georges, P.C.; Miller, W.J.; Meaney, D.F.; Sawyer, E.S.; Janmey, P.A. Matrices with compliance comparable to that of brain tissue select neuronal over glial growth in mixed cortical cultures. Biophys. J. 2006, 90, 3012-3018.
(40) Aurand, E.R.; Wagner, J.; Lanning, C.; Bjugstad, K.B. Building Biocompatible Hydrogels for Tissue Engineering of the Brain and Spinal Cord. J. Funct. Biomater., 2012, 3, 839-863.

(41) Thomas, R.C.; Chung, P.E.; Modi, S.P.; Hardy, J.G.; Schmidt, C.E. Sacrificial crystal templating of hyaluronic acidbased hydrogels. DOI:10.1016/j.eurpolymj.2016.10.022.

(42) Baier Leach, J.; Bivens, K.A.; Patrick, C.W.Jr.; Schmidt, C.E. Photocrosslinked hyaluronic acid hydrogels: natural, biodegradable tissue engineering scaffolds. Biotechnol. Bioeng. 2003, $82,578-89$.

(43) Drury, J.L.; Mooney, D.J. Hydrogels for tissue engineering: scaffold design variables and applications. Biomaterials, 2003, 24, 4337-4351.

(44) Garbay, B.; Heape, A.M.; Sargueil, F.; Cassagne, C. Myelin synthesis in the peripheral nervous system. Prog. Neurobiol. 2000, 61, 267-304.

(45) Lumelsky, N.L., Commentary: engineering of tissue healing and regeneration. Tissue Eng. 2007, 13, 1393-1398.

(46) Atala, A.; Irvine, D.J.; Moses, M.; Shaunak, S. Wound Healing Versus Regeneration: Role of the Tissue Environment in Regenerative Medicine. MRS Bulletin. 2010, 35, 597-6o6.

(47) Biernaskie, J.; Sparling, J.S.; Liu, J.; Shannon, C.P.; Plemel, J.R.; Xie, Y.; Miller, F.D.; Tetzlaff, W. Skin-derived precursors generate myelinating Schwann cells that promote remyelination and functional recovery after contusion spinal cord injury. J. Neurosci. 2007, 27, 9545-9559.

(48) Zhao, F.; Ma, M.L.; Xu, B. Molecular hydrogels of therapeutic agents. Chem. Soc. Rev. 2009, 38, 883-891. 


\section{Sacrificial crystal templated hydrogel preparation process}

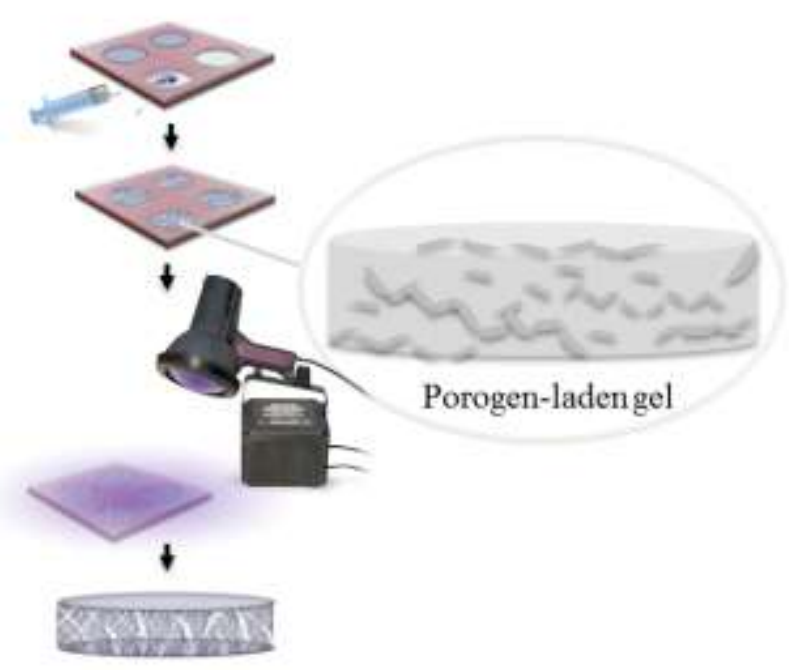

Table of Contents artwork 